\title{
Working memory load improves early stages of independent visual processing
}

\author{
Luca Cocchi $^{\mathrm{a}, *}$, Ulrike Toepel ${ }^{\mathrm{b}, \mathrm{c}}$, Marzia De Lucia ${ }^{\mathrm{b}, \mathrm{d}}$, Roberto Martuzzi ${ }^{\mathrm{e}}$, Stephen J. Wood ${ }^{\mathrm{a}}$, \\ Olivia Carter ${ }^{\mathrm{f}}$, Micah M. Murray ${ }^{\mathrm{b}, \mathrm{c}, \mathrm{d}}$ \\ a Melbourne Neuropsychiatry Centre, Department of Psychiatry, The University of Melbourne and Melbourne Health, Australia \\ ${ }^{\mathrm{b}}$ Radiology Department, Centre Hospitalier Universitaire Vaudois and University of Lausanne, Switzerland \\ ${ }^{c}$ Neuropsychology and Neurorehabilitation Service, Department of Clinical Neurosciences, Centre Hospitalier Universitaire Vaudois and University of Lausanne, Switzerland \\ d EEG Brain Mapping Core, Center for Biomedical Imaging of Lausanne and Geneva, Switzerland \\ e Ecole Polytechnique Federale Lausanne, Switzerland \\ ${ }^{\mathrm{f}}$ Department of Psychology, The University of Melbourne, Australia
}

\section{A R T I C L E I N F O}

\section{Article history:}

Received 2 June 2010

Received in revised form 9 October 2010

Accepted 15 October 2010

Available online 23 October 2010

\section{Keywords:}

Perception

Top-down

EEG

Working memory

Cognitive control

Dual-task

\begin{abstract}
A B S T R A C T
Increasing evidence suggests that working memory and perceptual processes are dynamically interrelated due to modulating activity in overlapping brain networks. However, the direct influence of working memory on the spatio-temporal brain dynamics of behaviorally relevant intervening information remains unclear. To investigate this issue, subjects performed a visual proximity grid perception task under three different visual-spatial working memory (VSWM) load conditions. VSWM load was manipulated by asking subjects to memorize the spatial locations of 6 or 3 disks. The grid was always presented between the encoding and recognition of the disk pattern. As a baseline condition, grid stimuli were presented without a VSWM context. VSWM load altered both perceptual performance and neural networks active during intervening grid encoding. Participants performed faster and more accurately on a challenging perceptual task under high VSWM load as compared to the low load and the baseline condition. Visual evoked potential (VEP) analyses identified changes in the configuration of the underlying sources in one particular period occurring $160-190 \mathrm{~ms}$ post-stimulus onset. Source analyses further showed an occipitoparietal down-regulation concurrent to the increased involvement of temporal and frontal resources in the high VSWM context. Together, these data suggest that cognitive control mechanisms supporting working memory may selectively enhance concurrent visual processing related to an independent goal. More broadly, our findings are in line with theoretical models implicating the engagement of frontal regions in synchronizing and optimizing mnemonic and perceptual resources towards multiple goals.
\end{abstract}

(c) 2010 Elsevier Ltd. All rights reserved.

\section{Introduction}

We live in a world that is rich in visual information. In order to succeed in this environment, we have developed the ability to maintain a representation of behaviorally relevant information for short periods while simultaneously processing new visual input. This short-term storage and maintenance of visual information is termed "working memory" (Baddeley, 1992, 2003) and is believed to involve a distributed network, encompassing prefrontal (Petrides, 2005; Petrides, Alivisatos, \& Frey, 2002) parietal (Todd \& Marois, 2004) and medial temporal (Axmacher et al., 2007; Axmacher, Schmitz, Weinreich, Elger, \& Fell, 2008; Rissman, Gazzaley, \& D’Esposito, 2008) brain regions.

\footnotetext{
* Corresponding author at: Melbourne Neuropsychiatry Centre, Alan Gilbert Building. University of Melbourne, 3053 Melbourne, VIC, Australia. Tel.: +6138344 1861; fax: +61393480469.

E-mail addresses: Icocchi@unimelb.edu.au, Icocchi78@gamil.com (L. Cocchi).
}

It is well established that occipital and parietal regions are involved in encoding visual information, whereas prefrontal regions appear to be responsible for maintaining this information for a short period of time (Funahashi, Bruce, \& Goldman-Rakic, 1993). However, it has been shown that the opposite is also true, with neurons in prefrontal regions encoding visual information (i.e., spatial location; Everling, Tinsley, Gaffan, \& Duncan, 2006; Funahashi, Bruce, \& Goldman-Rakic, 1990; Johnston \& Everling, 2006) and visual areas showing delay-period activity during a working memory task (Freedman, Riesenhuber, Poggio, \& Miller, 2003; Funahashi et al., 1990; Haxby, Petit, Ungerleider, \& Courtney, 2000; Pessoa, Gutierrez, Bandettini, \& Ungerleider, 2002; Postle, Druzgal, \& D’Esposito, 2003; Zaksas \& Pasternak, 2006). This knowledge has been recently extended by functional neuroimaging studies showing that low-level regions involved in visual information processing, such as the primary visual area (V1), may also play an important role in the online maintenance of information (Harrison \& Tong, 2009; Serences, Ester, Vogel, \& Awh, 2009; Todd \& Marois, 2004; Vogel \& Machizawa, 2004). 
Despite recent progress, the dynamic interplay between working memory and perceptual processes remains elusive. There is, however, increasing evidence suggesting that brain regions involved in working memory (i.e., prefrontal and parietal regions) impose top-down modulations on visual perceptual brain regions in line with task goals (Eger, Henson, Driver, \& Dolan, 2007; Egner \& Hirsch, 2005; Gazzaley et al., 2007; Johnston \& Everling, 2006; Postle, 2005). Recent dual-task experiments, mainly focusing on the effect of working memory load on selective attention, have been used to demonstrate a close and dynamic functional link between working memory and selective attention (Downing, 2000; Kim, Kim, \& Chun, 2005; Lavie \& De Fockert, 2005; Lavie, Hirst, de Fockert, \& Viding, 2004; Park, Kim, \& Chun, 2007; Woodman \& Luck, 2004; for a review see Awh, Vogel, \& Oh, 2006). Specifically, it has been shown that working memory load can interfere with the processing of information related to a secondary task (Kim et al., 2005; Park et al., 2007). While these interactions can either facilitate or impair performance on a secondary task, these effects can be best understood in terms of reduced processing of stimulus elements sharing overlapping resources with the working memory load.

Functional magnetic resonance imaging (fMRI) studies adopting similar dual-task designs (de Fockert, Rees, Frith, \& Lavie, 2001; Rissman, Gazzaley, \& D’Esposito, 2009) further showed that top-down signals related to working memory may amplify neuronal responses associated with a perceptual task and suppress task-irrelevant neural responses. However, under both visual and auditory high working memory load it was found that there was reduction in the brain's capacity to selectively suppress activity devoted to the processing of irrelevant visual information. By varying the visibility of the task-irrelevant background on which target working memory stimuli were presented, a related neuroimaging study showed a general deterioration of visual processing associated with the encoding of a visual background under high working memory load (2-back vs. 1-back conditions; Rose, Schmid, Winzen, Sommer, \& Buchel, 2005, see also Klemen, Buchel, Buhler, Menz, \& Rose, 2010). In keeping with the above, this result suggests that increased working memory load can reduce both attentional and perceptual resources available to a secondary task. These data are also consistent with the general proposition of the dual-task literature, suggesting that the saturation of the capacity-limited cognitive control (or "central executive") system induces a functional cost to the secondary task (Brisson \& Jolicoeur, 2007a, 2007b, 2007c; Pashler, 1994; Ruthruff, Pashler, \& Klaassen, 2001).

Electroencephalographic (EEG) investigations point to similar goal-directed modulations of early visual evoked potential (VEP) components associated with perceptual processing (P1 and N1) by working memory (Gazzaley, Cooney, McEvoy, Knight, \& D'Esposito, 2005; Holmes, Vuilleumier, \& Eimer, 2003; Morgan, Klein, Boehm, Shapiro, \& Linden, 2008; SanMiguel, Corral, \& Escera, 2008; Sreenivasan, Katz, \& Jha, 2007) and visual-spatial attention (Hillyard \& Anllo-Vento, 1998; Luck \& Hillyard, 1995). These top-down influences may indeed increase the processing speed of relevant information (Gazzaley et al., 2005) and/or lead to an amplification of attention-related activity (Hillyard \& Anllo-Vento, 1998). Moreover, the later phase of the novelty-P300 component associated with involuntary stimuli-driven attention (Escera, Alho, Winkler, \& Naatanen, 1998) was found to be decreased in amplitude under high working memory load (Morgan et al., 2008; SanMiguel et al., 2008; Shucard, Tekok-Kilic, Shiels, \& Shucard, 2009). In task involving the concurrent presentation of task-irrelevant distractors during working memory maintenance, this finding has been interpreted in terms of a reduced impact of distractors on maintained information (SanMiguel et al., 2008), highlighting the importance of goal-directed cognitive control mechanisms in the allocation of attentional and perceptual resources. Finally, VEP modulations have been observed within the first $100 \mathrm{~ms}$ of stimulus presentation that appear to reflect the specific nature of the working memory requirements - i.e., encoding of a novel cues for later recall as compared to the recognition of previously presented stimuli (Miller, Deouell, Dam, Knight, \& D’Esposito, 2008). These results are consistent with proposal that working memory function can influence even the earliest stages of visual processing.

Together, the abovementioned data suggest that temporary changes in working memory processes may have an important role in modulating perceptual processing. To date, research on the effect of working memory on perceptual processes has been somewhat limited to comparisons between the encoding of relevant $v$ s. irrelevant information (e.g., de Fockert et al., 2001; Gazzaley et al., 2005; Rissman et al., 2009; Rose et al., 2005; SanMiguel et al., 2008). It is unclear, however, whether perceptual processing is effected or impaired in situations where basic stimuli unrelated to a primary working memory task need to be encoded to achieve an independent secondary goal. To investigate this important question, we performed two experiments using a dual-task paradigm where subjects performed a basic visual perception task under three different visual-spatial working memory (VSWM) load conditions. In Experiment 1 , we investigated the effect of VSWM load on perceptual performance during the dual-task paradigm. We found perceptual encoding to be substantially facilitated under high VSWM load conditions. To characterize the neurophysiologic mechanisms underlying this unexpected behavioral effect, and more broadly the interaction between working memory and perceptual processing in this task context, Experiment 2 included VEP measurements and is accompanied by electrical neuroimaging analyses and source estimations (Murray, Brunet, \& Michel, 2008).

\section{Materials and methods}

\subsection{Participants}

Thirteen right-handed individuals (8 females) aged 23-39 years (mean $\pm \mathrm{SD}=29 \pm 4.6$ years) participated in Experiment 1 . A distinct group of twelve right-handed individuals took part in Experiment 2. Data analyses on Experiment 2 were conducted on eleven ( 3 female) right-handed individuals, aged from 25 to 41 years (mean $\pm \mathrm{SD}=30.4 \pm 4.8$ years). Data from one participant were excluded from the analysis due to excessive eye movement artefacts. None of the participants reported history of neurological or psychiatric illnesses, and all had normal or corrected to normal vision. The experimental protocol was approved by the Ethics Committee of the Faculty of Biology and Medicine at the Centre Hospitalier Universitaire Vaudois (CHUV) and University of Lausanne, and the Mental Health Research and Ethics Committee (MHREC; Melbourne, Australia).

\subsection{Stimulus presentation}

Details of the presentation procedure for the Experiment 1 are depicted in Fig. 1. In an initial VSWM encoding phase, 6 (high VSWM load), 3 (low VSWM load) or 0 black disks (baseline) were presented against a grey background. Participants viewed the display on a $12^{\prime \prime}$ PC monitor from a distance of $60 \mathrm{~cm}$. Each of the disks had a diameter of $1 \mathrm{~cm}$ (subtending $\sim 0.95^{\circ}$ visual angle) and were presented randomly and non-overlapping within $90 \%$ of the screen dimensions. This feature was adopted to ensure a similar attentional focus across conditions. During the VSWM encoding phase, the disks were presented for $1500 \mathrm{~ms}$ and then removed. After a period of $4500 \mathrm{~ms}$ the disks were presented again for $1500 \mathrm{~ms}$ during the recognition phase. On $50 \%$ of the trials, 3 disks were displaced $\left(\sim 2.0^{\circ}\right.$ visual angle) in a random direction from their original location during the encoding phase.

For the perceptual task, adapted from Kurylo, Pasternak, Silipo, Javitt, and Butler (2007), subjects were presented with a grid $\left(\sim 12.0^{\circ}\right.$ visual angle $)$ of 432 identical black squares $\left(6 \times 6\right.$ pixels, $\sim 0.02^{\circ}$ visual angle per square). The perceptual proximity task was chosen on the basis of previous studies showing that the discrimination of rows and columns relies on early and low-level visual processes that are predominantly (but not exclusively) subserved by the striate and extrastriate visual cortex (Singer, 1995; Stettler, Das, Bennett, \& Gilbert, 2002). This visual perceptual proximity task was presented, on a grey background, for $500 \mathrm{~ms}$ between the VSWM encoding and recognition phase $(2000 \mathrm{~ms}$ after the removal of the first presentation of the disks) in the high and low VSWM conditions, or was presented in the absence of any VSWM stimuli to form a baseline condition.

In Experiment 1, participants performed a difficult and an easy version of the perceptual task. In the difficult version of the task, the difference between the vertical and horizontal separation between the squares was minimal with a separation of 20 or 15 pixels $\left(\sim 0.5^{\circ}\right.$ or $\sim 0.38^{\circ}$ visual angle), respectively. Under these conditions 


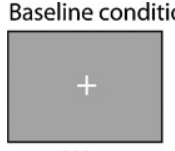

$500 \mathrm{~ms}$

White fix.cross

Low VSWM load condition

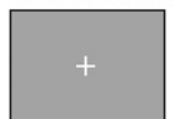

$500 \mathrm{~ms}$

White fix.cross

High VSWM load condition

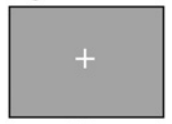

$500 \mathrm{~ms}$

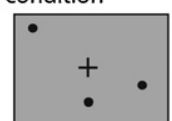

$1500 \mathrm{~ms}$

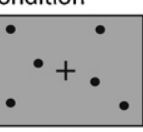

$1500 \mathrm{~ms}$

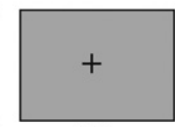

$2000 \mathrm{~ms}$

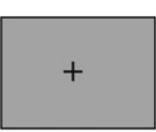

$2000 \mathrm{~ms}$

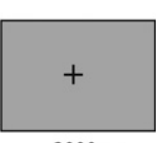

$2000 \mathrm{~ms}$

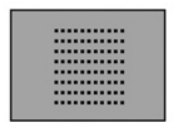

$500 \mathrm{~ms}$

Perception task

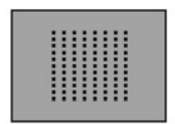

$500 \mathrm{~ms}$ Perception task

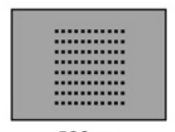

$500 \mathrm{~ms}$

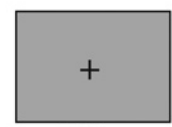

$2000 \mathrm{~ms}$

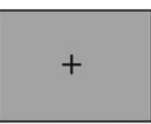

$2000 \mathrm{~ms}$
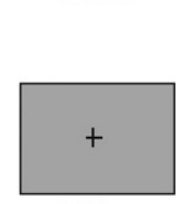

$2000 \mathrm{~ms}$

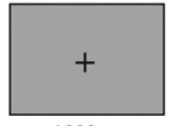

$1000 \mathrm{~ms}$

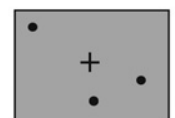

$1500 \mathrm{~ms}$ VSWM recognition

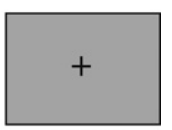

$1000 \mathrm{~ms}$
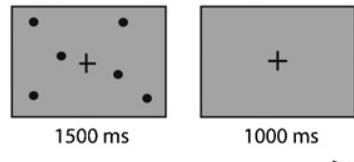

Time

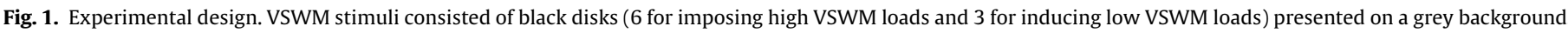

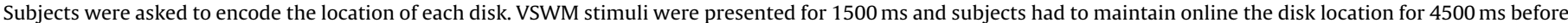

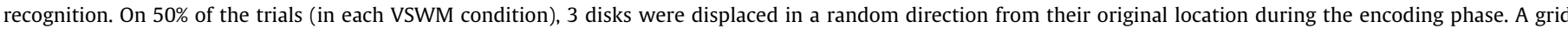

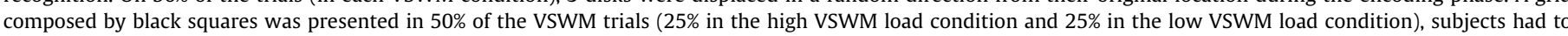

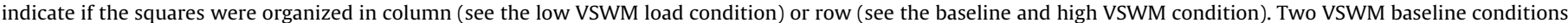
(i.e., without grid presentation during the delay period; not presented in the figure) were also presented to balance the design.

participants reported a very subtle, but detectable appearance of grouping of the squares as rows (horizontal lines) or columns (vertical lines) accordingly to the pure distance law (Kubovy \& van den Berg, 2008). In the easy version of the task, the relative difference between the vertical or horizontal separation between the squares was greatly increased to either 50 or 15 pixels $\left(\sim 1.26^{\circ}\right.$ or $\sim 0.38^{\circ}$ visual angle $)$ respectively. Under these conditions participants reported an easily detectable separation of the grid into rows or columns. In both conditions the arrangement of the grid as columns and rows was randomly assigned with equal probability across trials.

To test the respective effect of the VSWM load on the perception task, 5 trials conditions were used: (1) high VSWM load (six disk locations to be remembered)+ perceptual grid task; (2) low VSWM load (three disk locations to be remembered) + perceptual task grid; (3) perceptual grid task alone (no concurrent VSWM task); (4) high VSWM load alone (no concurrent perceptual task); (5) low VSWM load alone (no concurrent perceptual task). In Experiment 1, participants completed one block comprising 50 trials each (10 trials for each condition). The order of trials was randomized and the bidirectional association of the respective conditions within the block was counterbalanced. The trial sequences were created using a custom made MATLAB (MathWorks ${ }^{\mathrm{TM}}$ ) algorithm and the stimuli were generated with the Psychtoolbox 2.54 for MATLAB (http://psychtoolbox.org).

For Experiment 2 a slightly modified version of the difficult perceptual task was used. Participants viewed the display on a $21^{\prime \prime}$ PC monitor from a distance of $80 \mathrm{~cm}$. Because only correct trials are considered in the EEG analyses, a slightly larger square separation was used in order to reach an accuracy rate of approximately $90 \%$ - i.e., the vertical and horizontal separation between squares was either 22 or 15 pixels $\left(\sim 0.42^{\circ}\right.$ or $\sim 0.28^{\circ}$ visual angle), respectively. In Experiment 2 , subjects performed 12 blocks comprising 50 trials each ( 10 trials for each condition). In order maximize the number of trials obtained but minimize the total duration of testing time to avoid excessive subject fatigue, we reduced the length of the delay between the disks presentation to $3000 \mathrm{~ms}$ (this change did not affect the duration of the perceptual stimulus presentation). Overall, participants completed 120 trials per condition during an experimental session that lasted approximately $90 \mathrm{~min}$.

\subsection{Procedure}

In both experiments, participants were told that they were required to perform two separate unrelated tasks. In some trials they would be presented an array of disks and that in such trials they would have to memorize the location of each disk during this first "encoding" presentation (Fig. 1). The importance of remembering the exact location of each disk was emphasized by explaining to subjects that in some trials only one disk could move. Later during these trials they would be presented with a second array of disks for the "recognition" phase and their task was to determine whether any of the disks had changed location. In some trials they would be presented with a grid of small squares and would be asked to judge whether it was arranged in columns or rows.

It was clearly explained to participants that in some trials they would either be presented: (1) the disk memory task alone; (2) the grid perception task alone or (3) both tasks, with the grid perception stimulus being presented between the first and second presentation of the disks (in the middle of the VSWM task). In all trials in which the grid was presented, participants were asked to indicate, via key press, whether they perceived the small squares to be arranged in columns (index finger) or rows (middle finger) - as quickly and accurately as possible after the grid presentation. In trials in which the disk memory task was presented, participants were asked to indicate whether the disk locations were the same (index finger) or different (middle finger) - as quickly and accurately as possible after the second disk presentation. All subjects performed a short practice session before performing in the actual experiment to ensure that they had understood the procedure and could perform the task with the desired accuracy (i.e., around 80, 90 and $>95 \%$, depending on the task version). Subjects were instructed to view the VSWM and grid stimuli without moving their eyes to avoid ocular artifacts. For this purpose, a fixation cross was presented in the middle of the screen throughout each trial. This fixation cross disappeared when the perceptual stimulus (grid) was presented in the middle of the screen (see Fig. 1).

\subsection{Electrophysiological recordings}

In Experiment 2, continuous EEG was acquired at $1024 \mathrm{~Hz}$ through a 160 channel Biosemi ActiveTwo system (Biosemi, Amsterdam, Netherlands) referenced to the CMS-DRL ground, which functions as a feedback loop driving the average potential across the electrode montage to the amplifier zero. The EEG was online band-pass filtered between 0.1 and $100 \mathrm{~Hz}$. Recordings took place in an electrically shielded sound-attenuated cabin.

\subsection{Behavioral and EEG analyses}

\subsubsection{Behavioral analyses}

Accuracy and reaction times (RTs) related to perceptual and VSWM tasks were analyzed using standard parametric approaches [within-subjects analysis of variance (ANOVA) and paired $t$-tests]. The normal distribution of the data was controlled beforehand by one-sample Kolmogorov-Smirnov test. Mauchly's test was used to control for violations of sphericity in the within-subjects ANOVAs. Analyses were performed using the IBM SPSS Statistics 18 software (http://www.spss.com/software/statistics/). Partial $\eta^{2}$ and Cohen's $d$ were additionally estimated to assess the size of the observed effects. We utilized signal detection measures [ $d$-prime $\left(d^{\prime}\right)$; Macmillan \& Creelman, 2005] to confirm changes in perceptual sensitivity as a function of VSWM load. Finally, post hoc statistical power analyses were calculated using the software $G^{*}$ Power (version 3.1.2; Faul, Erdfelder, Lang, \& Buchner, 2007). This analysis, computed using standardized effect size (Cohen's $d$ and partial $\eta^{2}$ ) along with the sample size, was performed to evaluate the possibility of type II error.

\subsubsection{EEG analyses}

The EEG analyses were conducted using the Cartool software (http://brainmapping.unige.ch/cartool.php) and additional customized MATLAB scripts. Epochs of EEG recordings over the -100 to $500 \mathrm{~ms}$ period relative to the onset of the visual grid were averaged for each correctly answered trial and each subject. EEG epochs with ocular artifacts were semi-automatically identified and rejected using horizontal and vertical electrooculogram and a rejection criterion of $\pm 80 \mu \mathrm{V}$ at all electrodes. Data from artifact electrodes were interpolated using 
3-dimensional splines (Perrin, Pernier, Bertrand, Giard, \& Echallier, 1987). Data were band-pass filtered from 0.1 to $40 \mathrm{~Hz}$, including a $50 \mathrm{~Hz}$ notch filter. Groupaveraged VEPs were computed as a function of whether the grid was preceded by a high VSWM load, low VSWM load or no VSWM load (baseline condition). Data were recalculated to the common average reference prior to group-averaging, and baseline correction was applied to the $100 \mathrm{~ms}$ epoch preceding the grid stimulus.

2.5.2.1. General analysis strategy. Electrophysiological analyses were conducted on local and global measures of the electric field at the scalp. The latter of these so-called electrical neuroimaging analyses - enable the differentiation of effects following from modulations in the strength of responses of statistically indistinguishable brain generators from alterations in the configuration of these generators (viz. the topography of the electric field at the scalp). These methods have been shown to be useful for analyzing EEG data from larger electrode sensor arrays and have been extensively detailed elsewhere (Murray et al., 2008 for a methods tutorial), so that only essential details related to the current experiment are provided here.

2.5.2.2. VEP waveform modulations. We analyzed VEP waveform data millisecondwise from all electrodes over the post-stimulus period in an ANOVA with the factor condition (no, low or high VSWM load). Temporal auto-correlation was corrected through the application of a $20 \mathrm{~ms}$ contiguous temporal criterion for effect persistence (Guthrie \& Buchwald, 1991). This criterion was also applied to the analysis of Global Field Power and the topographic measures described below. The results of the analyses are presented as an intensity plot representing time (post-grid onset), electrode location, and $p$-values $(\leq 0.05)$ at each data point. Note that no correction was applied for multiple testing across the electrodes. This analysis provides an initial visual impression of the effects. However, analyses of voltage VEP waveforms are reference-dependent, such that the statistical outcome (as well as the time series of the VEP itself) will change with the choice of a different reference site (c.f., Fig. 1 in Murray et al., 2008; also Lehmann, 1987). As such, voltage waveform analyses by themselves comport limited interpretational power either from a statistical or neurophysiologic standpoint (Dien \& Santuzzi, 2005). Consequently, and given our interest in determining the effect of VSWM load on the active network of brain regions during the perceptual task, our analyses focus on global measures of the electric field at the scalp, which are reference-independent and consider data from the entire electrode montage.

2.5.2.3. Global electric field analyses. The electrical neuroimaging analyses of the VEPs focused on two features of the electric field. The first type of analysis identified periods of time when the topography of the VEP differed within and across experimental conditions, irrespective of the strength of the VEP. Changes in the electric field topography forcibly derive from changes in the configuration of intracrania sources (though the converse need not forcibly be true) (Lehmann, 1987). The second type of analysis identified periods of time when the strength of the VEP differed across experimental conditions, irrespective of the topography of the VEP. The reader should note that these two dependent measures are independent of each other.

There were two objectives with the topographic analyses. The first was to determine the sequence of VEP topographies within the response to each experimental condition. For readers less familiar with these methods, this can be loosely equated to determining the "components" of the VEP from each experimental condition, where a VEP "component" is defined by its latency and scalp distribution (i.e. topography) (e.g., Clark, Fan, \& Hillyard, 1995). It has recently been shown that results of the present topographic analyses are highly similar to those obtained using independent component analysis (ICA), despite differences in theoretical bases and assumptions regarding the data (De Lucia, Michel, \& Murray, 2010) The second, parallel objective was to determine if and when the VEP topographies differed between conditions. These objectives were addressed through an atomize and agglomerate hierarchical clustering (AAHC) of the group-average VEPs concatenated across the three experimental conditions (i.e., three $600 \mathrm{~ms}$ VEPs, totaling $1800 \mathrm{~ms}$ of data). Here we provide an overview, but full details can be found in published tutorials (Murray et al., 2008).

In the AAHC, each time point (i.e., each millisecond of the VEP) can be represented as a vector with dimensionality equal to the number of electrodes. Each of these time points from the concatenated dataset is initially designated as a unique cluster. From all of these clusters, the worst is identified as that with the lowes Global Explained Variance (see Murray et al., 2008 for formula), which is a metric of how well a given cluster describes the whole dataset. This worst cluster is then atomized, so that the data from each time point contributing to the worst cluster is re-ascribed to the other cluster(s) with which it best correlates spatially. For each cluster the centroid is calculated, which is the mathematical average across time points when a given cluster had the highest spatial correlation. This clustering and identification of the worst cluster is iterated until there is only 1 remaining cluster. Thus, for a given set of $n$ clusters, the experimenter can visualize what its topographic distribution looks like. These topographic distributions are colloquially referred to as template maps. The reader should note, that the cluster analysis does not including the relative timing of a data point in its attribution of a given template map. Thus, any temporal structure is an inherent property of the ERP and is not induced by the clustering analysis.
The next step is the determination of how many different template maps adequately describe the concatenated group-averaged VEPs from the different experimental conditions. Again, using the more colloquial term "component", the next step is to determine how many different components are present in the concatenated VEPs. This also serves as a hypothesis generation tool in that the experimenter has a qualitative description of whether and when all experimental conditions appear to be described by the same or different clusters/components. Typically, there are periods when the same template map is observed in all experimental conditions and other periods when one template map is observed for one experimental condition and another (or several others) for another experimental condition. The optimal number of template maps is determined using the combination of a cross-validation criterion and Krzanowski-Lai criterion (see formulae in Murray et al., 2008). This combination identifies the best trade-off between describing the greatest variance in the data with as few template maps as possible.

The pattern of template maps observed in the group-averaged VEPs is then tested in the single-subject VEPs. That is, for a given time period the template maps observed in the group-averaged VEPs are compared with the VEPs from singlesubjects in response to each experimental condition. This comparison is based on the calculation of spatial correlation, which is directly related to global dissimilarity (formula in Murray et al., 2008). From this calculation over a given time period, the experimenter obtains a measure of how well a given template map describes the VEP from each experimental condition (on average across subjects) and how well another template map describes the VEP from each experimental condition. This procedure is colloquially referred to as "fitting"; the output of which is submitted to repeated measures ANOVA using factors of experimental condition and template map. In the event of a significant interaction, it can be concluded that different template maps - i.e., VEP topographies - better account for different experimental conditions. This would constitute evidence that different conditions, at a given latency, are engaging different configurations of intracranial sources (albeit potentially overlapping to a large degree).

A complementary assessment of whether the topography of the VEP significantly differs across experimental conditions is based on the calculation of Global Dissimilarity (Lehmann \& Skrandies, 1980; see Murray et al., 2008 for recent publication of formula), which is the root mean square of the difference between two strength-normalized electric fields (here VEP topography across conditions at a given instant in time). Global Dissimilarity can range from 0 to 2, with the former in the case of identical topographic distributions and the latter in the case of inverted topographic distributions. The analysis of Global Dissimilarity is based on randomization tests (i.e., within-subject permutations of the data across conditions using Monte Carlo bootstrapping) and is colloquially referred to as topographic ANOVA (TANOVA), though we would emphasize that no actual ANOVA is used here. In the case of the present study, the single-subject VEPs of all three experimental conditions were first subjected to a randomization procedure (Koenig, Melie-Garcia, Stein, Strik, \& Lehmann, 2008; Wirth et al., 2008) with 5000 repetitions to test against the null-hypothesis that electric field topographies evoked by each condition are not different from the mean topography across all conditions. Next, TANOVAs between pairs of experimental conditions were performed. Only differences meeting the statistical threshold of $p \leq 0.05$ for at least 20 ms contiguously were considered as reliable.

Analysis of the strength of the VEP irrespective of its topography was accomplished using Global Field Power (GFP; Lehmann \& Skrandies, 1980). GFP is calculated as the square root of the mean of the squared value recorded at each electrode in the 160-channel montage ( $v s$. the average reference) and represents the spatial standard deviation of the electric field at the scalp, yielding larger values for stronger electric fields. In a similar manner to what is described above to analyses the VEP voltage waveforms, we conducted a millisecond-wise ANOVA on GFP waveforms. However, the reader should note that GFP is a reference-independent measure. Analyses of GFP are also independent to those of topography as described above. We emphasize that the observation of a GFP modulation in the absence of a topographic modulation would most parsimoniously be interpreted as a strength or power modulation of statistically indistinguishable neural generators across experimental conditions.

2.5.2.4. Source estimations. We estimated the sources in the brain underlying the VEPs from each condition using a distributed linear inverse solution (ELECTRA) applying the local autoregressive average (LAURA) regularization approach to address the non-uniqueness of the inverse problem (Grave de Peralta Menendez, Gonzalez Andino, Lantz, Michel, \& Landis, 2001). The inverse solution algorithm is based on biophysical principles derived from the quasi-static Maxwell's equations; most notably the fact that independent of the volume conductor model used to describe the head only irrotational and not solenoidal currents contribute to the EEG (Grave de Peralta Menendez et al., 2001; Grave de Peralta Menendez, Murray, Michel, Martuzzi, \& Gonzalez Andino, 2004). Uniqueness of the inverse problem requires the application of a regularization strategy. In our study, homogenous regression coefficients in all directions and within the whole solution space were used. The current implementation of LAURA was generated with the Spherical Model with Anatomical Constraints (SMAC; Spinelli, Andino, Lantz, Seeck, \& Michel, 2000). LAURA uses a realistic head model, and the solution space included 3005 nodes, equally distributed within the gray matter of the Montreal Neurological Institute's average brain. Prior basic and clinical research has documented and discussed in 
detail the spatial accuracy of this inverse solution (e.g., Gonzalez Andino, Michel, Thut, Landis, \& Grave de Peralta, 2005; Gonzalez Andino, Murray, Foxe, \& de Peralta Menendez, 2005; Grave de Peralta Menendez et al., 2001; Michel et al., 2004). As an output, LAURA provides current density measures, the scalar values of which can be statistically analyzed at each node in the solution space. Time periods for which intracranial sources were estimated and statistically compared between conditions were defined by the results of the topographic cluster analysis described above. Statistical analyses of source estimations were performed by first averaging the VEP data over such time periods to generate a single data point per period for each participant and condition. The inverse solution ( 11 participants $\times 3$ conditions) was then estimated for each of the 3005 nodes. A one-way ANOVA with condition as the within-subjects factor was calculated at each node using the variance across participants, the result of this analysis was used as an inclusive mask for post hoc paired $t$-tests. In both analyses only nodes with $p$-values $\leq 0.05\left(t_{(10)} \geq 2.23\right)$ and clusters of at least 20 contiguous nodes were considered reliable. This spatial criterion was determined using the AlphaSim program (available at http://afni.nimh.nih.gov). The 10,000 Monte Carlo permutations performed on our lead field matrix revealed a false positive probability of $<0.005$ for a cluster greater than 14 nodes. The results of the source estimations were rendered on the MNI brain with the Talairach and Tournoux (1988) coordinates of the largest statistical differences indicated.

\section{Results}

\subsection{Experiment 1}

\subsubsection{Difficult perception task}

Accuracy and RTs of the correct trials in the visual proximity task (grid perception) significantly differed between the three VSWM load contexts. Within-subjects ANOVA identified a significant main effect of VSWM load for both accuracy $\left(F_{(2,24)}=3.31, p=0.05\right.$; partial $\left.\eta^{2}=0.22\right)$ and RTs $\left(F_{(2,24)}=3.34, p=0.05\right.$; partial $\left.\eta^{2}=0.22\right)$. Participants were more accurate in the visual proximity task in the context of a high VSWM load (88\%) as opposed to no load (80\%; $t_{(12)}=2.99, p=0.01$; Cohen's $\left.d=0.48\right)$. No significant differences were found between high $v s$. low (85\% correct) load $\left(t_{(12)}=1.00\right.$, $p=0.33 ; d=0.20)$ or low load $v s$. baseline $\left(t_{(12)}=1.38, p=0.19\right.$; $d=0.28)$ conditions. Perceptual sensitivity measures $\left(d^{\prime}\right)$ confirmed these results by showing an overall difference between conditions (high load: mean \pm s.e.m $d^{\prime}=3.1 \pm 0.45$; low load $d^{\prime}=2.6 \pm 0.47$ and baseline $d^{\prime}=2.3 \pm 0.44 ; F_{(2,24)}=3.82, p=0.03$; partial $\left.\eta^{2}=0.24\right)$. Post hoc analyses showed a greater perceptual sensitivity of participants in the high VSWM load condition compared to baseline $\left(t_{(12)}=2.94\right.$, $p=0.01 ; d=0.48)$. RTs in the perceptual task were faster in the high VSWM load condition (mean \pm s.e.m.: $1041 \pm 58 \mathrm{~ms}$ ) as opposed to baseline $\left(1126 \pm 67 \mathrm{~ms}, t_{(12)}=2.49, p=0.02 ; d=0.37\right.$ ). Participants also tended to respond faster in the high VSWM load condition compared to the low VSWM condition ( $1083 \pm 62 \mathrm{~ms})$ although this result did not reach significance $\left(t_{(12)}=1.95, p=0.07 ; d=0.19\right)$.

With respect to the performance in the working memory task (i.e., disk pattern recognition), a within-subjects two-way ANOVA revealed a significant effect of condition (with vs. without intervening perceptual task; $F_{(1,12)}=16.26, p<0.01$; partial $\left.\eta^{2}=0.57\right)$ but no effect of VSWM load $\left(F_{(1,12)}=1.73, p=0.21\right.$; partial $\eta^{2}=0.12$, accuracy above $85 \%$ in both conditions) and no condition $\times$ load interaction $\left(F_{(1,12)}=1.08, p=0.31\right.$; partial $\left.\eta^{2}=0.08\right)$. Post hoc analyses indicated a negative impact of the concurrent perceptual task on disk pattern recognition $\left(t_{(25)}=4.15, p<0.01\right.$; $d=1.14)$. Analyses on RTs showed a significant main effect of VSWM load $\left(F_{(1,12)}=9.19, p=0.01 ; \eta^{2}=0.43\right)$, but no effect of condition $\left(F_{(1,12)}=1.16, p=0.30 ; \eta^{2}=0.08\right)$ and no interaction between condition and load $\left(F_{(1,12)}=0.001, p=0.97 ; \eta^{2}<0.01\right)$. Post hoc analyses indicated that participants were slower in the high VSWM load conditions compared to the low VSWM load conditions $\left(t_{(25)}=2.36\right.$, $p=0.02 ; d=0.34$ ).

\subsubsection{Easy perception task}

Accuracy in the easy grid perception task was above $97 \%$ under each working memory load $\left(F_{(2,24)}=0.76, p=0.47\right.$; partial $\eta^{2}=0.06$; power $=0.44$ ). Contrary to the results obtained for the difficult grid perception task, RTs on the correct trials did not differ significantly between the three conditions $\left(F_{(2,24)}=2.23\right.$, $p=0.12$; partial $\eta^{2}=0.15$; power $=0.88$ ). Although the high statistical power supports the absence of RT differences, the pattern of RTs appears opposite to those observed in the difficult task version (i.e., high VSWM load mean \pm s.e.m.: $906 \pm 37 \mathrm{~ms}$, low VSWM load $879 \pm 41 \mathrm{~ms}$ and baseline $859 \pm 42 \mathrm{~ms}$ conditions). A withinsubjects two-way ANOVA confirmed that the impact of VSWM load on RTs differed significantly between the difficult and easy grid perception task (interaction of condition $\mathrm{x}$ task: $F_{(2,24)}=5.90, p<0.01$; partial $\eta^{2}=0.33$ ). This result indicates that the increased RT speed obtained under high VSWM load in the difficult task version does not reflect a generalized effect of VSWM on the facilitation of motor responses.

In accordance with the performance in the working memory task in the difficult grid perception version, accuracy rates showed a significant effect of condition $\left(F_{(1,12)}=12.71, p<0.01 ; \eta^{2}=0.51\right)$ but no effect of load $\left(F_{(1,12)}=0.12, p=0.73 ; \eta^{2}=0.01\right)$ and no condition $x$ load interaction $\left(F_{(1,12)}=1.44, p=0.25 ; \eta^{2}=0.10\right)$. Post hoc analyses indicated a negative impact of the concurrent perceptual task on working memory performance $\left(t_{(25)}=2.83, p<0.01\right.$; $d=0.74)$. Analyses on RTs showed a significant effect of condition $\left(F_{(1,12)}=10.92, p<0.01 ; \eta^{2}=0.47\right)$, but no effect of $\operatorname{load}\left(F_{(1,12)}=2.81\right.$, $\left.p=0.11 ; \eta^{2}=0.19\right)$ and no interaction between load and condition $\left(F_{(1,12)}=2.17, p=0.16 ; \eta^{2}=0.15\right)$. Post hoc analyses indicated a negative impact of the concurrent perceptual task on reaction times in the working memory task $\left(t_{(25)}=2.83, p<0.01 ; d=0.37\right)$.

\subsection{Experiment 2}

\subsubsection{Behavioral results}

As expected from Experiment 1, accuracy rates in the visual proximity task (grid perception) were high irrespective of concurrent VSWM load $(\sim 90 \%)$ and did not differ significantly between conditions $\left(F_{(2,20)}=1.00, p=0.38\right.$; partial $\eta^{2}=0.09$; power $=0.55$; $d^{\prime} F_{(2,20)}=1.03, p=0.37$; partial $\eta^{2}=0.09$; power $=0.55$ ). A withinsubjects ANOVA with RTs from the correct trials showed a significant effect of VSWM load $\left(F_{(2,20)}=7.14, p<0.01\right.$; partial $\left.\eta^{2}=0.41\right)$. In accordance with the results obtained in the difficult proximity task in Experiment 1, participants were faster in the context of a high VSWM load (mean \pm s.e.m.: $871 \pm 44 \mathrm{~ms}$ ) as opposed to either a low VSWM load $\left(890 \pm 43 \mathrm{~ms} ; t_{(10)}=2.32, p=0.04\right.$; $d=0.13)$ or no VSWM load $\left(908 \pm 47 \mathrm{~ms} ; t_{(10)}=3.55, p<0.01\right.$; $d=0.24)$. No significant RT differences were observed between the low VSWM load and the no load baseline condition $\left(t_{(10)}=1.70\right.$, $p=0.11 ; d=0.12$ ). Importantly, despite the relative ease of the perceptual task, high VSWM load still provides a significant facilitation to the perception of visual proximity.

In agreement with the performance in the working memory task obtained in the difficult task version of Experiment 1, a withinsubjects two-way ANOVA showed a significant main effect of condition $\left(F_{(1,10)}=53.25, p<0.01 ; \eta^{2}=0.84\right)$, but no effect of VSWM $\operatorname{load}\left(F_{(1,10)}=0.77, p=0.40 ; \eta^{2}=0.07\right)$ and no load $\times$ condition interaction $\left(F_{(1,10)}=2.17, p=0.17 ; \eta^{2}=0.18\right)$. Post hoc analyses indicated a negative impact of the concurrent perceptual task on VSWM maintenance $\left(t_{(21)}=6.38, p<0.01 ; d=1.20\right)$. Analysis of RTs showed significant main effects of load $\left(F_{(1,10)}=20.58, p=0.01 ; \eta^{2}=0.67\right)$ and condition $\left(F_{(1,10)}=10.59, p=0.09 ; \eta^{2}=0.51\right)$ as well as an interaction between load and condition $\left(F_{(1,10)}=9.58, p=0.01\right.$; $\eta^{2}=0.49$ ). Participants were slower in the high VSWM load conditions compared to the low VSWM load conditions $\left(t_{(21)}=4.18\right.$, $p<0.01 ; d=0.43$ ), and the perceptual task had a negative impact on VSWM maintenance $\left(t_{(21)}=3.61, p<0.01 ; d=0.45\right)$. Further, analyses showed that increase in RT from low to high VSWM load conditions was greater when the perceptual task was not presented 
Waveforms and electrode-wise analysis

A VEP modulations on one representative electrode

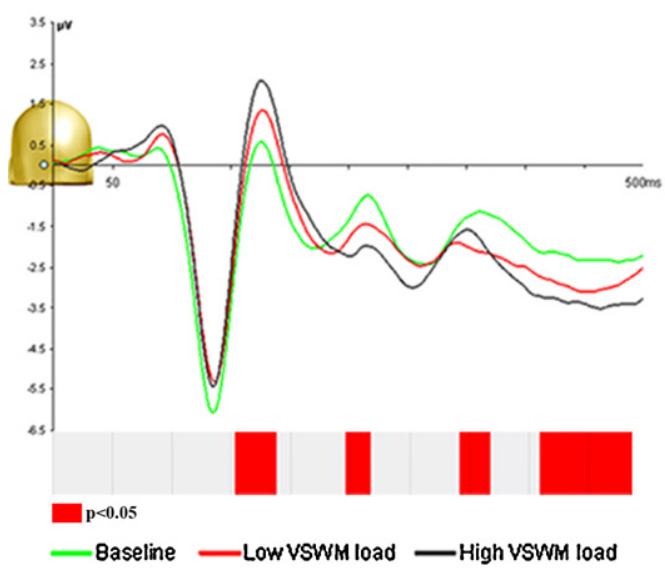

B Time point-wise ANOVA contrasting the three perceptual conditions

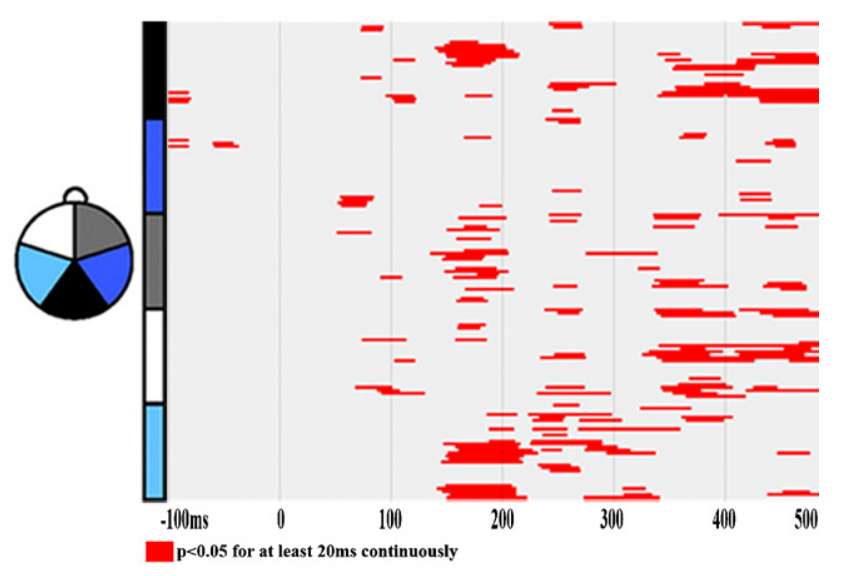

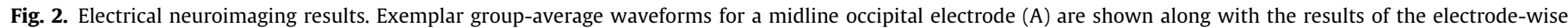
ANOVA over the post-grid period between the three conditions (B). Spatially widespread and temporally sustained differences began at 160 ms post stimulus onset.

compared to when the grid was concurrently presented $\left(t_{(10)}=3.09\right.$, $p=0.01 ; d=1.23$.

\subsubsection{Electrophysiological results}

VEP waveforms from an exemplar posterior midline scalp site are shown in Fig. 2A. Voltage waveform data from each scalp electrode were first analyzed in a millisecond-wise ANOVA. Temporally sustained effects of condition were observed at posterior electrodes between 150 and $200 \mathrm{~ms}, 230-270 \mathrm{~ms}$ and $330 \mathrm{~ms}$ onwards (see Fig. 2B). Voltage waveforms analysis provides a first level of evidence regarding the time periods of differences between the three experimental conditions. However, because this analysis is impacted by the reference choice (even in the case of the average reference), we focus instead on reference-independent measures of the global electric field at the scalp.

The group-averaged VEPs were subjected to a common hierarchical topographic cluster analysis (detailed in Section 2). Nine template maps were identified over the post-stimulus period from all experimental conditions, explaining $94.99 \%$ of the variance of the concatenated group-average VEP dataset. While over most of the post-stimulus period there was one template map that accounted for responses from all conditions at a given latency, during the 160-190 ms period two template maps were identified that differentially accounted for the group-average VEPs (Fig. 3A). This pattern was then tested in the fitting procedure and within-subjects ANOVA.

There was a significant condition $\times$ map presence interaction over the $160-190 \mathrm{~ms}$ post-stimulus interval $\left(F_{(2,20)}=4.057, p=0.03\right.$; $\eta^{2}=0.29$ ). Post hoc analyses indicated that the top map depicted in Fig. 3A (blue frame) best characterized subjects' responses when perceiving the grid under low $\left(t_{(10)}=2.53, p=0.03 ; d=0.67\right)$ and high VSWM loads $\left(t_{(10)}=2.32, p=0.04 ; d=0.79\right)$ as opposed to the baseline condition. No significant differences were observed between the high and low VSWM loads $\left(t_{(10)}=0.71, p=0.48\right.$; $d=0.19$ ). As the single-subject responses are forcibly ascribed to either one template map during the fitting procedure, equiopposite results were observed for the bottom map (green frame, see Fig. 3B).

In order to determine whether the observed topographic clusters indeed differed statistically in terms of spatial configuration, we further analyzed the Global Dissimilarity (Lehmann \& Skrandies, 1980) across conditions on a millisecond-wise basis. The results of these analyses are displayed in Supplementary Fig. 1A. The topographic randomization procedure (querying whether the electric field topography under each load condition is different from the mean topography across all three load conditions) indicated an

\section{A. Hierarchical clustering analysis}

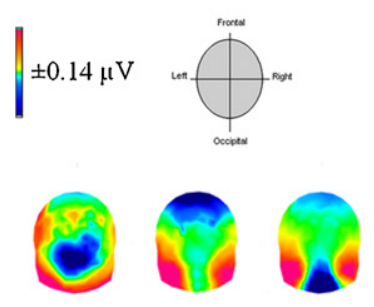

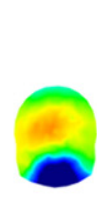
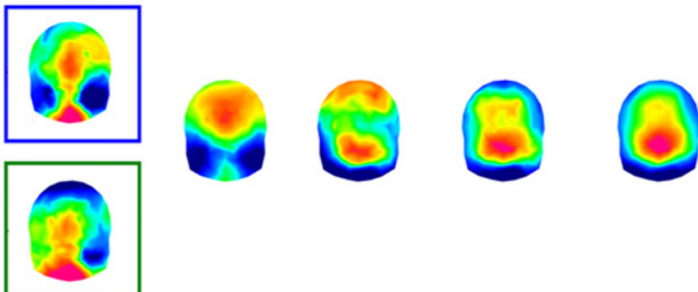

415.500

\section{B. Spatial correlation 'fitting'}

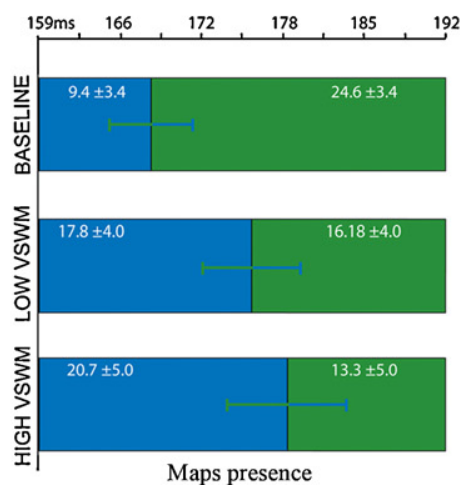

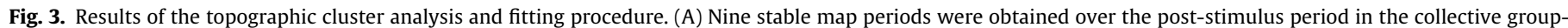

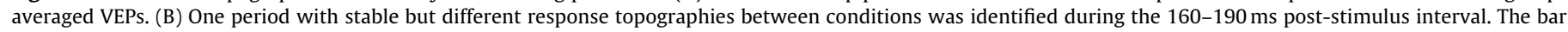

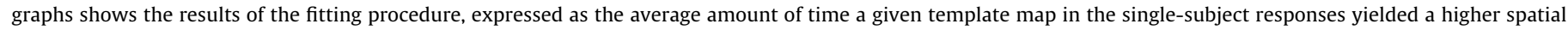
correlation with the group-wise results than an alternative one ( \pm s.e.m.). 
effect of VSWM load between 160 and $200 \mathrm{~ms}$. Post hoc analyses between pairs of conditions revealed that the topographic modulations during this time period were driven by the dissimilarity in spatial configuration of the electric fields under the high VSWM load $v$ s. baseline condition, and low VSWM load vs. baseline condition. There was no evidence of topographic differences between high and low VSWM loads. Independent from modulations in response topography, the VEPs were also analyzed in terms of response strength modulations, quantified by Global Field Power (GFP; Lehmann \& Skrandies, 1980). Supplementary Fig. 1B illustrates the results of a time point-wise ANOVA on the GFP including all conditions. The analysis revealed an effect of VSWM load on global response strength only between 440 and 500 ms, thus substantially later than the above topographic differences.

Taken together, the VEP analyses based on global measures of the electric field at the scalp indicate that working memoryinduced effects on the visual proximity task manifested in topographic modulations during relatively early stages of visual processing (starting at $\sim 160 \mathrm{~ms}$ post-stimulus onset) across conditions. These effects were due to the differential treatment of VSWM load conditions as opposed to the baseline condition. These topographic changes are indicative of varying configurations of neural generators during the visual proximity task irrespective of the amount of VSWM load.

LAURA was used to estimate the neural generators underlying the VEP responses over the $160-190 \mathrm{~ms}$ period. These were computed separately for each subject and load condition and were extracted as scalar values at each of the 3005 node within the solution space. Group-average source estimations for each condition over the 160-190 ms post-stimulus period are illustrated in Fig. 4. Distributed sources were evident in occipital, parietal and posterior

\section{LAURA source estimation (160-190ms)}
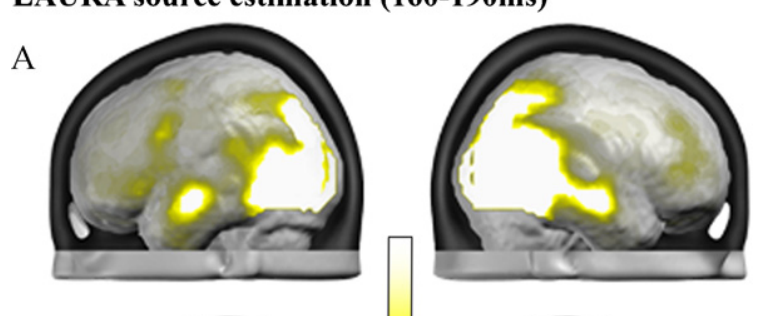

$\mathrm{B}$
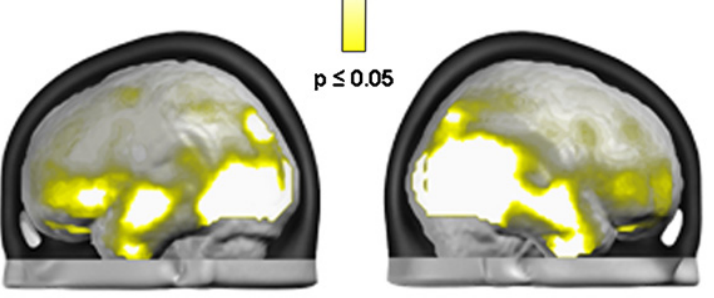

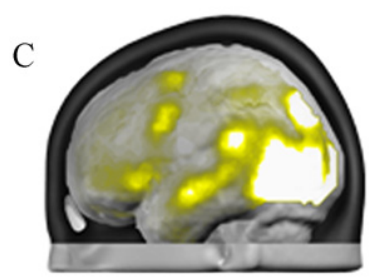

Left

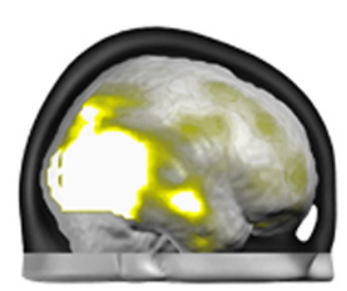

Right
Fig. 4. Source estimations. Functional neuronal network maps associated to the early period of topographical differences (160-190 ms post stimulus onset). (A) perception of the grid without VSWM load (baseline condition); (B) perception of the grid when concurrently maintaining the past sensory representation of six visualized disks (high VSWM load); (C) perception of the grid when concurrently maintaining the past sensory representation of three visualized disks (low VSWM load). Increased involvement of fronto-temporal regions during the grid perception is evident in the high VSWM load context vs. baseline (see B vs. A).

\section{LAURA source estimation (160-190ms)}
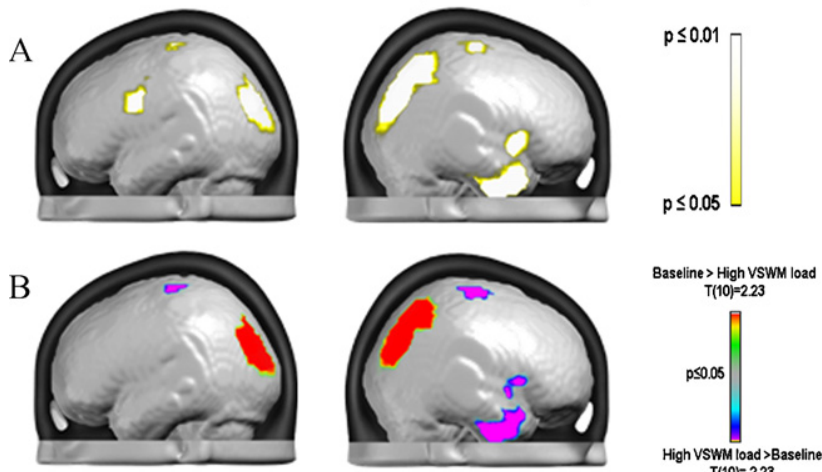

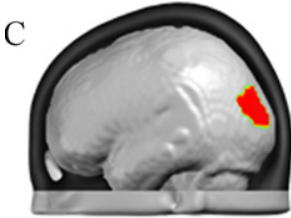

Left

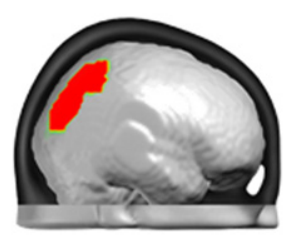

Right

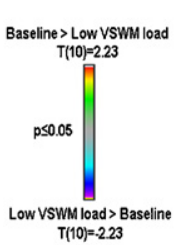

Fig. 5. Differences in the functional network maps over the $160-190 \mathrm{~ms}$ post stimulus onset period between: (A) regions found to be differentially engaged between conditions when analyzed using ANOVA; (B) $t$-test contrast between neural sources active under high VSWM load $v$ s. baseline condition. Right inferior and dorsal frontal regions as well as anterior temporal regions were significantly more activated under high VSWM load while bilateral occipito-parietal sources where stronger active under baseline load; (C) $t$-test contrast between neural sources active under low VSWM load $v$ s. baseline condition. Only increased bilateral occipito-parietal activity was observed under baseline condition but no extra sources for low VSWM load.

temporal regions during grid perception under either VSWM load. Descriptively, the functional network during grid perception in the working memory load contexts (either high or low) extended more anteriorly - i.e., involving anterior temporal and frontal sources in addition to those mentioned above.

Source estimations were first evaluated with a $1 \times 3$ ANOVA utilizing the scalar values obtained at each of the 3005 solution points for all three conditions ( $p<0.05 ; K_{E}=20$ adjacent nodes). Results of this ANOVA are shown in Fig. $5 \mathrm{~A}$ and indicate differences in frontal, anterior temporal and parieto-occipital cortices. To detail the networks specifically active for each condition, post hoc paired $t$-tests between the source estimation to high VSWM load vs. baseline and low load $v s$. baseline were additionally conducted. When statistically comparing the neural sources active during the high VSWM load $v s$. baseline, parts of the parietal and occipital cortices (bilaterally) were found to be more active under the baseline condition. In contrast, grid perception under the high VSWM load led to stronger responses within dorsal and ventral frontal regions as well as the right anterior temporal lobe (Fig. 5B). When contrasting the neural sources between low VSWM load vs. baseline, parts of the superior parietal and occipital lobe revealed stronger activations under the baseline condition. There was no evidence that the low VSWM load resulted in stronger activity vs. baseline (Fig. 5C). Table 1 provides an overview of the clusters. The reported coordinates of maximal $t$-values within a functional cluster are based on the system of Talairach and Tournoux (1988).

\section{Discussion}

To our knowledge this is the first study to investigate the effect of increasing working memory load on the processing of concurrent visual information that is behaviorally relevant but completely independent of the working memory task. To our surprise, high VSWM load was found to improve performance on the percep- 
Table 1

Source estimation (160-190 ms).

\begin{tabular}{|c|c|c|c|}
\hline Region with Brodmann area (BA) indicated & Hemisphere (cluster dimension) & Maximal $T$ value & Coordinates $(X, Y, Z)$ \\
\hline \multicolumn{4}{|l|}{ Baseline $>$ high VSWM } \\
\hline Occipital cortex (BA 19) & $\mathrm{L}(78)$ & 5.45 & $-33,-87,24$ \\
\hline Parietal cortex (BA 7) & $\mathrm{R}(85)$ & 4.64 & $9,-64,41$ \\
\hline \multicolumn{4}{|l|}{ Baseline > low VSWM } \\
\hline Occipital cortex (BA 18) & $\mathrm{L}(28)$ & 3.78 & $-21,-94,19$ \\
\hline Parietal cortex (BA 7) & $\mathrm{R}(83)$ & 6.12 & $9,-65,54$ \\
\hline \multicolumn{4}{|l|}{ High VSWM > baseline } \\
\hline Dorsomedial frontal cortex (BA 6) & $\mathrm{R}(26)$ & 6.30 & $3,-15,63$ \\
\hline Inferior frontal gyrus (BA 45) & $\mathrm{R}(72)$ & 2.83 & $50,13,3$ \\
\hline
\end{tabular}

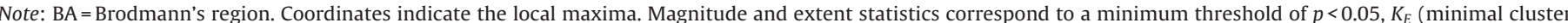

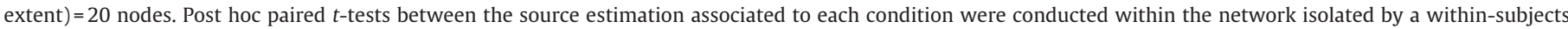
ANOVA ( $p<0.05, K_{E}=20$ nodes $)$.

tual task. Electrical neuroimaging analyses of VEP data indicated that these improvements were accompanied by topographic modulations during the $160-190 \mathrm{~ms}$ post-stimulus period (i.e., the N1 component). While there was no evidence for effects of VSWM load on the very initial stages of sensory-perceptual processing, effects at this latency are nonetheless occurring during relatively early stages of visual processing. Overall, the nature of the observed functional modulations highlights the importance of the dynamic interplay between perceptual and VSWM mechanisms in optimizing the management (i.e., encoding and maintenance) of relevant information in challenging multi-goal contexts.

Data from our first set of behavioral tests indicated that high VSWM load increased both the speed and the accuracy of a visual perceptual task relative to either a low load or the absence of VSWM requirements (i.e., baseline condition). Results from the easy version of the perception task provide compelling evidence that such facilitation is due to a direct modulation of perceptual processes by VSWM load and not to changes in later non-perceptual processes, such as decision-making and motor plan execution. Indeed, when the separation of the vertical and horizontal arrangement of the grid was very large and clearly detectible, reaction time in the perceptual task did not significantly change as a function of VSWM load. In fact, in the easy version of the perceptual task, the effect of VSWM load on reaction time appears to be reversed with high VSWM load leading to slower RTs.

While perceptual facilitation in high VSWM load contexts is reminiscent of earlier studies showing improved performance in visual discrimination tasks when attention is covertly directed to the stimulus location prior to its presentation (Downing, 1988; Posner, Snyder, \& Davidson, 1980), pure attention modulations seem unlikely to explain the observed effect. Reaction times between high and low VSWM load conditions differed, although the stimulus onset, and therefore the level of anticipation, was identical between the two conditions. Furthermore, the absence of the working memory stimulus during the baseline trials should have acted as a cue for participants to prepare for the consecutive perceptual task. Therefore, if anticipation was relevant to the performance on the perceptual task, one would expect that performance would have been best in the single-task baseline condition; here we saw the opposite pattern of results. Moreover, data from the dual-task literature generally indicate that presentation of two tasks simultaneously, or in close succession, leads to an increase in reaction time for the second task (Pashler, 1994; Ruthruff et al., 2001). This effect is explained by the suggestion that cognitive control processes are not allocated uniformly between concurrent tasks but serially to each task. Our results appear in contrast with these findings. Indeed, although non-perceptual processes may become slower, perceptual processes appear enhanced in the high working memory load condition. A possible explanation for this apparent contradiction is that in our task context, strong demands on working memory resources lead to functional changes selectively facilitating the exe- cution of the concurrent visual perceptual task. This hypothesis is in line with the EEG data discussed below.

Neurophysiologically, working memory load induced differences in the spatio-temporal VEP response pattern related to the grid perception. Indeed, local electrode and global topographic analyses consistently revealed an influence of working memory load on the VEP between 160 and $190 \mathrm{~ms}$ after the onset of the perceptual stimulus (i.e., grid). These topographic differences are indicative of, at least partially, non-overlapping neural source configurations accompanying the grid encoding under concurrent working memory demands as opposed to no-load conditions (i.e., baseline). Notably, the topographic changes in the VEPs were observed in the absence of (quantitative) modulations of the global response strength over the same time period. Thus, in line with interpretation of the behavioral findings, the topographic changes in VEP responses are hard to explain in terms of a pure attention-driven interference of VSWM load on visual grid encoding mechanisms. This also reduces the likelihood that, given the constant inter-stimuli delay, hypothetical subject's anticipation interfered with the first volley of top-down signals on perceptual processes. As previously shown, such attention interference would rather manifest in quantitative VEP modulations i.e., response strength differences (Hillyard \& Anllo-Vento, 1998; Luck, Woodman, \& Vogel, 2000). However, such strength modulations were only observed much later ( $\sim 40 \mathrm{~ms}$ post-grid onset), some $300 \mathrm{~ms}$ after the first working memory-induced topographic modulation, and probably point to VSWM interference on later processes including attention, perceptual decision-making and action selection.

Distributed source estimations over the time period showing working memory-induced topographic differences (160-190 ms) support the involvement of partially overlapping, but nonetheless distinguishable, brain networks during grid encoding under concurrent VSWM demands vs. no working memory load. Although the VEP topographic clustering failed to identify significantly differing topographic maps for grid perception under high vs. low working memory load conditions, source estimations were found to differ between these conditions. One possibility that will need to be further evaluated is that source estimations may provide (at least in some instances) improved sensitivity to focal changes in brain activity compared to analyses of the electrode data themselves (Britz, Landis, \& Michel, 2009). This proposition is somehow supported by behavioral data presented here, showing a significant modulation of perceptual processes only under high VSWM load.

Together, results from the VEP topographic clustering and the neural source estimations indicate that the perception of the grid in high and low VSWM load trials was associated with a significant reduction of occipito-parietal activity compared to the grid perception in baseline condition trials (Fig. 5). This robust finding suggests that VSWM demands down-regulate neural activity in brain regions involved in visual-spatial encoding. This result is 
generally consistent with data from dual-task experiments showing that concurrent processing of a first target stimulus interferes with perceptual processing of a second visual target- i.e., amplitude reduction of the second half $(170-190 \mathrm{~ms})$ of the occipital N1 component (Brisson \& Jolicoeur, 2007a). The proposition of an occipito-parietal down-regulation is also in line with results showing that high working memory load prevents the encoding of irrelevant information during the presentation of working memory related stimuli (Rose et al., 2005) or task-irrelevant stimuli that participants are explicitly asked to ignore (SanMiguel et al., 2008). This seems to suggest a general decrease of high-order resources allocated to "working memory-irrelevant" attentional and perceptual processes as a function of working memory load (see also Klemen et al., 2010; Spinks, Zhang, Fox, Gao, \& Hai Tan, 2004). Paradoxically, in our task context, high VSWM load enhanced perceptual processing of behaviorally relevant information that is independent from the working-memory task. This apparent incongruence may be explained by an active perceptual gating for goal-relevant information provoked by a saturation of working memory resources. Top-down modulation of occipito-parietal activity during grid perception in the VSWM load conditions vs. baseline may therefore occur in the context of a general reduction of sensory-perceptual processes induced by the need to optimize scarce executive resources (Postle, 2005). This hypothesis is supported by behavioral data obtained in Experiment 2, showing impaired working memory performance as a function of load, at least in term of reaction time, in trials without the perceptual task compared to trials with the perceptual task. Moreover, these findings seem to exclude the possibility that enhanced perceptual performance in the high VSWM load condition were due to an increased allocation of working memory resources to perceptual processes. While our results indicate improved perceptual performance, they are not in disagreement with the dual-task literature that involved tasks with distinct cognitive and motor demands that were not as relevant in the current study. Future research is needed to elucidate the limits of the perceptual facilitation observed here.

Reduced occipito-parietal activity could only be statistically confirmed under low VSWM load. However, the observation that grid perception under high VSWM load ( $v s$. baseline) induced concurrent significant changes in frontal and temporal sources as well, suggest that the recruitment of additional fronto-temporal regions during grid encoding under high VSWM load may also contribute to the reliable behavioral advantage in the perceptual task. It has been shown that frontal (Petrides, 2005; Rissman et al., 2008) as well as temporal (Axmacher et al., 2007; Rissman et al., 2008) regions play a critical role in the online maintenance and storing of sensory representations. The increased involvement of temporal regions for maintaining behaviorally relevant information under high VSWM load has previously been related to increased frontotemporal contributions following the saturation of the limited capacity occipito-parietal working memory system and the need for online information storage that is resistant to fluctuations in attention and sensory distractors (Rissman et al., 2008). Moreover, elevated activation of right inferior and dorsal frontal regions has been associated with different dimensions of cognitive control such as stimulus recognition and online maintenance, perceptual encoding modulation, attention and inhibitory control, response conflict processes and decision making (Anderson, Mannan, Rees, Sumner, \& Kennard, 2009; Benetti et al., 2009; Bunge, Ochsner, Desmond, Glover, \& Gabrieli, 2001; Dux, Ivanoff, Asplund, \& Marois, 2006; Ekstrom, Roelfsema, Arsenault, Bonmassar, \& Vanduffel, 2008; Hampshire, Chamberlain, Monti, Duncan, \& Owen, 2010; Hedden \& Gabrieli, 2006; for reviews see Badre, 2008; Fuster, 2001). Consistent with these reports, data from our study support the hypothesis of increased contribution of frontal mechanisms to coordinate the encoding of new relevant information with the maintenance of past sensory representations in challenging multi-task contexts. More broadly, our data are in agreement with theoretical models suggesting a key role of frontal/prefrontal brain regions in temporally synchronizing and optimizing mnemonic and perceptual processes according to behavioral goals (Miller \& Cohen, 2001; see also Badre, 2008).

To the best of your knowledge, results from this set of experiments provide the first evidence of improved performance on a perceptual task that is behaviorally relevant but independent from a concurrent working memory task. Electrical neuroimaging analyses suggests that the enhancement of perceptual performence in high working memory load conditions may be related to a direct modulation of brain regions involved in sensory-perceptual processes by frontal mechanisms supporting cognitive control. Further studies using similar dual-task paradigms and assessing changes in effective functional connectivity between frontal and regions involved in perceptual processes are, however, required to confirm this hypothesis and better understand the dynamic interplay between working memory and concurrent processes involved in stimuli encoding - i.e., perceptual, attention, decision-making and action selection. For example, one remaining question is whether the relationship between working memory and perceptual processes is linear or would follow an inverted U-shaped function with the effects reversing under sufficiently high working memory load. Studies systematically exploring the effect of different working memory loads on concurrent perceptual functions will provide important information on the functional interaction between these processes. Using task designs similar to the one employed here may also be relevant in understanding the altered interplay between cognitive and sensory processes in psychiatric conditions.

\section{Competing interest}

\section{None}

\section{Acknowledgements}

We thank Jean-Francois Knebel and Nathalie Bourquin for providing assistance during EEG recordings. Prof. Giuseppe Foffi (EPFL) helped in the development of the Matlab algorithm used to generate the stimuli presentation sequence. The Cartool software (http://brainmapping.unige.ch/Cartool.htm) has been programmed by Denis Brunet, from the Functional Brain Mapping Laboratory, Geneva, Switzerland, and is supported by the Center for Biomedical Imaging (CIBM) of Geneva and Lausanne. Financial support was provided by the Swiss National Science Foundation (PBLAB-119622, PBLAB3-119622 to LC), (K-33K1_122518/1 to $\mathrm{MDL}$ ), and (3100AO-118419 to MMM); and the Swiss Foundation for Fellowships in Biology and Medicine [PASMP3_129357/1 to LC]. SJW is supported by a Clinical Career Development Award from the NHMRC (Australia) and OC is supported by a Career Development Award from the NHMRC (Australia) 628590.

\section{Appendix A. Supplementary data}

Supplementary data associated with this article can be found, in the online version, at doi:10.1016/j.neuropsychologia.2010.10.021.

\section{References}

Anderson, E. J., Mannan, S. K., Rees, G., Sumner, P., \& Kennard, C. (2009). Overlapping functional anatomy for working memory and visual search. Experimental Brain Research, 200(1), 91-107.

Awh, E., Vogel, E. K., \& Oh, S. H. (2006). Interactions between attention and working memory. Neuroscience, 139(1), 201-208.

Axmacher, N., Mormann, F., Fernandez, G., Cohen, M. X., Elger, C. E., \& Fell, J. (2007). Sustained neural activity patterns during working memory in the human medial temporal lobe. Journal of Neuroscience, 27(29), 7807-7816. 
Axmacher, N., Schmitz, D. P., Weinreich, I., Elger, C. E., \& Fell, J. (2008), Interaction of working memory and long-term memory in the medial temporal lobe. Cerebral Cortex, 18(12), 2868-2878.

Baddeley, A. (1992). Working memory. Science, 255(5044), 556-559.

Baddeley, A. (2003). Working memory: Looking back and looking forward. Nature Review Neuroscience, 4(10), 829-839.

Badre, D. (2008). Cognitive control, hierarchy, and the rostro-caudal organization of the frontal lobes. Trends in Cognitive Sciences, 12(5), 193-200.

Benetti, S., Mechelli, A., Picchioni, M., Broome, M., Williams, S., \& McGuire, P. (2009). Functional integration between the posterior hippocampus and prefrontal cortex is impaired in both first episode schizophrenia and the at risk mental state. Brain, 132(9), 2426-2436.

Brisson, B., \& Jolicoeur, P. (2007a). Cross-modal multitasking processing deficits prior to the central bottleneck revealed by event-related potentials. Neuropsychologia, 45(13), 3038-3053.

Brisson, B., \& Jolicoeur, P. (2007b). Electrophysiological evidence of central interference in the control of visuospatial attention. Psychonomic Bulletin and Review, $14,126-132,1$

Brisson, B., \& Jolicoeur, P. (2007c). A psychological refractory period in access to visual short-term memory and the deployment of visual-spatial attention: Multitasking processing deficits revealed by event-related potentials. Psychophysiology, 44(2), 323-333.

Britz, J., Landis, T., \& Michel, C. M. (2009). Right parietal brain activity precedes perceptual alternation of bistable stimuli. Cerebral Cortex, 19(1) $55-65$.

Bunge, S. A., Ochsner, K. N., Desmond, J. E., Glover, G. H., \& Gabrieli, J. D. (2001) Prefrontal regions involved in keeping information in and out of mind. Brain 124(Pt 10), 2074-2086.

Clark, V. P., Fan, S., \& Hillyard, S. A. (1995). Identification of early visual evoked potential generators by retinotopic and topographic analyses. Human Brain Mapping $2,170-187$.

de Fockert, J. W., Rees, G., Frith, C. D., \& Lavie, N. (2001). The role of working memory in visual selective attention. Science, 291(5509), 1803-1806.

De Lucia, M., Michel, C. M., \& Murray, M. M. (2010). Comparing ICA-based and single trial topographic ERP analyses. Brain Topography, 23(2), 119-127.

Downing, C. J. (1988). Expectancy and visual-spatial attention: Effects on perceptual quality. Journal of Experimental Psychology: Human Perception and Performance $14(2), 188-202$

Dien, J. \& Santuzzi, A. M. (2005). Application of repeated measures ANOVA to highdensity ERP datasets: A review and tutorial. In T. C. Handy (Ed.), Event-related potentials: A methods handbook (pp. 57-82). Cambridge, MA: MIT Press.

Downing, P. E. (2000). Interactions between visual working memory and selective attention. Psychological Science, 11(6), 467-473.

Dux, P. E., Ivanoff, J., Asplund, C. L., \& Marois, R. (2006). Isolation of a centra bottleneck of information processing with time-resolved FMRI. Neuron, 52(6), 1109-1120.

Eger, E., Henson, R. N., Driver, J., \& Dolan, R. J. (2007). Mechanisms of top-down facilitation in perception of visual objects studied by FMRI. Cerebral Cortex, 17(9), 2123-2133.

Egner, T., \& Hirsch, J. (2005). Cognitive control mechanisms resolve conflict through cortical amplification of task-relevant information. Nature Neuroscience, 8(12) 1784-1790.

Ekstrom, L. B., Roelfsema, P. R., Arsenault, J. T., Bonmassar, G., \& Vanduffel, W. (2008) Bottom-up dependent gating of frontal signals in early visual cortex. Science, 321(5887), 414-417.

Escera, C., Alho, K., Winkler, I., \& Naatanen, R. (1998). Neural mechanisms of involuntary attention to acoustic novelty and change. Journal of Cognitive Neuroscience, 10(5), 590-604.

Everling, S., Tinsley, C. J., Gaffan, D., \& Duncan, J. (2006). Selective representation of task-relevant objects and locations in the monkey prefrontal cortex. European Journal of Neuroscience, 23(8), 2197-2214

Faul, F., Erdfelder, E., Lang, A. G., \& Buchner, A. (2007). G*Power 3: A flexible statistical power analysis program for the social, behavioral, and biomedical sciences. Behavioural Research Methods, 39(2), 175-191.

Freedman, D. J., Riesenhuber, M., Poggio, T., \& Miller, E. K. (2003). A comparison of primate prefrontal and inferior temporal cortices during visual categorization. Journal of Neuroscience, 23(12), 5235-5246.

Funahashi, S., Bruce, C. J., \& Goldman-Rakic, P. S. (1990). Visuospatial coding in primate prefrontal neurons revealed by oculomotor paradigms. Journal of $\mathrm{Neu}$ rophysiology, 63(4), 814-831.

Funahashi, S., Bruce, C. J., \& Goldman-Rakic, P. S. (1993). Dorsolateral prefrontal lesions and oculomotor delayed-response performance: Evidence for mnemonic "scotomas". Journal of Neuroscience, 13(4), 1479-1497.

Fuster,J. M. (2001). The prefrontal cortex - An update: Time is of the essence. Neuron $30(2), 319-333$

Gazzaley, A., Cooney, J. W., McEvoy, K., Knight, R. T., \& D’Esposito, M. (2005) Top-down enhancement and suppression of the magnitude and speed of neura activity. Journal of Cognitive Neuroscience, 17(3), 507-517.

Gazzaley, A., Rissman, J., Cooney, J., Rutman, A., Seibert, T., Clapp, W., et al. (2007) Functional interactions between prefrontal and visual association cortex contribute to top-down modulation of visual processing. Cerebral Cortex, 17(Suppl 1), i125-i135.

Gonzalez Andino, S. L., Michel, C. M., Thut, G., Landis, T., \& Grave de Peralta, R. (2005). Prediction of response speed by anticipatory high-frequency (gamm band) oscillations in the human brain. Human Brain Mapping, 24(1), 50-58.
Gonzalez Andino, S. L., Murray, M. M., Foxe, J. J., \& de Peralta Menendez, R. G. (2005). How single-trial electrical neuroimaging contributes to multisensory research. Experimental Brain Research, 166(3-4), 298-304.

Grave de Peralta Menendez, R., Gonzalez Andino, S., Lantz G., Michel, C. M., \& Landis, T. (2001). Noninvasive localization of electromagnetic epileptic activity I. Method descriptions and simulations. Brain Topography, 14(2), 131-137.

Grave de Peralta Menendez, R., Murray, M. M., Michel, C. M., Martuzzi, R., \& Gonzalez Andino, S. L. (2004). Electrical neuroimaging based on biophysical constraints. Neuroimage, 21(2), 527-539.

Guthrie, D., \& Buchwald, J. S. (1991). Significance testing of difference potentials. Psychophysiology, 28(2), 240-244.

Hampshire, A., Chamberlain, S. R., Monti, M. M., Duncan, J., \& Owen, A. M. (2010). The role of the right inferior frontal gyrus: Inhibition and attentional control. Neuroimage, 50(3), 1313-1319.

Harrison, S. A., \& Tong, F. (2009). Decoding reveals the contents of visual working memory in early visual areas. Nature, 458(7238), 632-635.

Haxby, J. V., Petit, L., Ungerleider, L. G., \& Courtney, S. M. (2000). Distinguishing the functional roles of multiple regions in distributed neural systems for visual working memory. Neuroimage, 11(5 Pt 1), 380-391.

Hedden, T., \& Gabrieli, J. D. (2006). The ebb and flow of attention in the human brain. Nature Neuroscience, 9(7), 863-865.

Hillyard, S. A., \& Anllo-Vento, L. (1998). Event-related brain potentials in the study of visual selective attention. Proceedings of the National Academy of Sciences USA 95(3), 781-787.

Holmes, A., Vuilleumier, P., \& Eimer, M. (2003). The processing of emotional facial expression is gated by spatial attention: Evidence from event-related brain potentials. Brain Research Cognitive Brain Research, 16(2), 174-184.

Johnston, K., \& Everling, S. (2006). Monkey dorsolateral prefrontal cortex sends task-selective signals directly to the superior colliculus. Journal of Neuroscience, 26(48), 12471-12478

Kim, S. Y., Kim, M. S., \& Chun, M. M. (2005). Concurrent working memory load can reduce distraction. Proceedings of the National Academy of Sciences USA, 102(45), 16524-16529.

Klemen, J., Buchel, C., Buhler, M., Menz, M. M., \& Rose, M. (2010). Auditory working memory load impairs visual ventral stream processing: Toward a unified model of attentional load. Journal of Cognitive Neuroscience, 22(3), 437-446.

Koenig, T., Melie-Garcia, L., Stein, M., Strik, W., \& Lehmann, C. (2008). Establishing correlations of scalp field maps with other experimental variables using covariance analysis and resampling methods. Clinical Neurophysiology, 119(6), $1262-1270$

Kubovy, M., \& van den Berg, M. (2008). The whole is equal to the sum of its parts: A probabilistic model of grouping by proximity and similarity in regular patterns. Psychological Review, 115(1), 131-154.

Kurylo, D. D., Pasternak, R., Silipo, G., Javitt, D. C., \& Butler, P. D. (2007). Perceptual organization by proximity and similarity in schizophrenia. Schizophrenia Research, 95(1-3), 205-214.

Lavie, N., \& De Fockert, J. (2005). The role of working memory in attentional capture. Psychonomic Bulletin and Review, 12(4), 669-674.

Lavie, N., Hirst, A., de Fockert, J. W., \& Viding, E. (2004). Load theory of selective attention and cognitive control. Journal of Experimantal Psychology: General, 133(3), 339-354.

Lehmann, D. (1987). Principles of spatial analysis. In A. S. Gevins, \& A. Remond (Eds.), Handbook of electroencephalography and clinical neurophysiology. Methods of analysis of brain electrical and magnetic signals (pp. 309-354). Amsterdam: Elsevier.

Lehmann, D., \& Skrandies, W. (1980). Reference-free identification of components of checkerboard-evoked multichannel potential fields. Electroencephalography Clinical Neurophysiology, 48(6), 609-621.

Luck, S. J., \& Hillyard, S. A. (1995). The role of attention in feature detection and conjunction discrimination: An electrophysiological analysis. International Journal of Neuroscience, 80(1-4), 281-297.

Luck, S. J., Woodman, G. F., \& Vogel, E. K. (2000). Event-related potential studies of attention. Trends in Cognitive Science, 4(11), 432-440.

Macmillan, N. A., \& Creelman, C. D. (2005). Detection Theory: A User's Guide (second ed.). Mahwah, New Jersey 07430: Lawrence Erlbaum Associates.

Michel, C. M., Murray, M. M., Lantz, G., Gonzalez, S., Spinelli, L., \& Grave de Peralta, R. (2004). EEG source imaging. Clinical Neurophysiology, 115(10), 2195-2222.

Miller, B. T., Deouell, L. Y., Dam, C., Knight, R. T., \& D’Esposito, M. (2008). Spatiotemporal dynamics of neural mechanisms underlying component operations in working memory. Brain Research, 1206, 61-75.

Miller, E. K., \& Cohen, J. D. (2001). An integrative theory of prefrontal cortex function. Annual Review in Neuroscience, 24, 167-202.

Morgan, H. M., Klein, C., Boehm, S. G., Shapiro, K. L., \& Linden, D. E. (2008). Working memory load for faces modulates P300, N170, and N250r. Journal of Cognitive Neuroscience, 20(6), 989-1002.

Murray, M. M, Brunet, D., \& Michel, C. M. (2008). Topographic ERP analyses: A stepby-step tutorial review. Brain Topography, 20(4), 249-264.

Park, S., Kim, M. S., \& Chun, M. M. (2007). Concurrent working memory load can facilitate selective attention: Evidence for specialized load. Journal of Experimental Psychology: Human Perception and Performance, 33(5), 1062-1075.

Pashler, H. (1994). Dual-task interference in simple tasks: Data and theory. Psychological Bullein, 116(2), 220-244.

Perrin, F., Pernier, J., Bertrand, O., Giard, M. H., \& Echallier, J. F. (1987). Mapping of scalp potentials by surface spline interpolation. Electroencephalography and Clinical Neurophysiology, 66(1), 75-81. 
Pessoa, L., Gutierrez, E., Bandettini, P., \& Ungerleider, L. (2002). Neural correlates of visual working memory: fMRI amplitude predicts task performance. Neuron, 35(5), 975-987.

Petrides, M. (2005). Lateral prefrontal cortex: Architectonic and functional organization. Philosophical Transactions of the Royal Society B: Biological Sciences, 360(1456), 781-795.

Petrides, M., Alivisatos, B., \& Frey, S. (2002). Differential activation of the human orbital, mid-ventrolateral, and mid-dorsolateral prefrontal cortex during the processing of visual stimuli. Proceedings of the National Academy of Sciences USA, 99(8), 5649-5654.

Posner, M. I., Snyder, C. R., \& Davidson, B. J. (1980). Attention and the detection of signals. Journal of Experimental Psychology, 109(2), 160-174.

Postle, B. R. (2005). Delay-period activity in the prefrontal cortex: One function is sensory gating. Journal of Cognitive Neuroscience, 17(11), 1679-1690.

Postle, B. R., Druzgal, T. J., \& D'Esposito, M. (2003). Seeking the neural substrates of visual working memory storage. Cortex, 39(4-5), 927-946.

Rissman, J., Gazzaley, A., \& D’Esposito, M. (2008). Dynamic adjustments in prefrontal, hippocampal, and inferior temporal interactions with increasing visual working memory load. Cerebral Cortex, 18(7), 1618-1629.

Rissman, J., Gazzaley, A., \& D'Esposito, M. (2009). The effect of non-visual working memory load on top-down modulation of visual processing. Neuropsychologia, 47(7), 1637-1646.

Rose, M., Schmid, C., Winzen, A., Sommer, T., \& Buchel, C. (2005). The functional and temporal characteristics of top-down modulation in visual selection. Cerebral Cortex, 15(9), 1290-1298.

Ruthruff, E., Pashler, H. E., \& Klaassen, A. (2001). Processing bottlenecks in dual-task performance: Structural limitation or strategic postponement? Psychonomic Bulletin and Review, 8(1), 73-80.

SanMiguel, I., Corral, M. J., \& Escera, C. (2008). When loading working memory reduces distraction: Behavioral and electrophysiological evidence from an auditory-visual distraction paradigm. Journal of Cognitive Neuroscience, 20(7), 1131-1145.

Serences, J. T., Ester, E. F., Vogel, E. K., \& Awh, E. (2009). Stimulus-specific delay activity in human primary visual cortex. Psychological Science, 20(2), 207-214.
Shucard, J. L., Tekok-Kilic, A., Shiels, K., \& Shucard, D. W. (2009). Stage and load effects on ERP topography during verbal and spatial working memory. Brain Research, $1254,49-62$.

Singer, W. (1995). Development and plasticity of cortical processing architectures. Science, 270(5237), 758-764.

Spinelli, L., Andino, S. G., Lantz, G., Seeck, M., \& Michel, C. M. (2000). Electromagnetic inverse solutions in anatomically constrained spherical head models. Brain Topography, 13(2), 115-125.

Spinks, J. A., Zhang, J. X., Fox, P. T., Gao, J. H., \& Hai Tan, L. (2004). More workload on the central executive of working memory, less attention capture by novel visual distractors: Evidence from an fMRI study. Neuroimage, 23(2), 517-524.

Sreenivasan, K. K., Katz, J., \& Jha, A. P. (2007). Temporal characteristics of top-down modulations during working memory maintenance: An event-related potential study of the N170 component. Journal of Cognitive Neuroscience, 19(11) 1836-1844.

Stettler, D. D., Das, A., Bennett, J., \& Gilbert, C. D. (2002). Lateral connectivity and contextual interactions in macaque primary visual cortex. Neuron, 36(4), 739-750.

(1988). Talairach, J., \& Tournoux, P. (Eds.), Co-planar stereotaxic atlas of the human brain: 3-Dimensional proportional system-An approach to cerebral imaging New York: Thieme Medical Publishers.

Todd, J. J., \& Marois, R. (2004). Capacity limit of visual short-term memory in human posterior parietal cortex. Nature, 428(6984), 751-754

Vogel, E. K., \& Machizawa, M. G. (2004). Neural activity predicts individual differences in visual working memory capacity. Nature, 428(6984), 748-751.

Wirth, M., Horn, H., Koenig, T., Razafimandimby, A., Stein, M., Mueller, T., et al. (2008) The early context effect reflects activity in the temporo-prefrontal semantic system: Evidence from electrical neuroimaging of abstract and concrete word reading. Neuroimage, $42(1)$, 423-436.

Woodman, G. F., \& Luck, S. J. (2004). Visual search is slowed when visuospatial working memory is occupied. Psychonomic Bulletin and Review, 11(2), 269-274.

Zaksas, D., \& Pasternak, T. (2006). Directional signals in the prefrontal cortex and in area MT during a working memory for visual motion task.Journal of Neuroscience, 26(45), 11726-11742. 